\title{
A TWO-ZONE POTENTIAL RELAXATION SCHEME FOR HANDLING GRID WIRES
}

\author{
R. 1. Smizh \\ S. L. Rompel
}

June 1, 1973

Prepared for U.S. Atomic Energy Commission under contracl No. W-7405-Eng-48

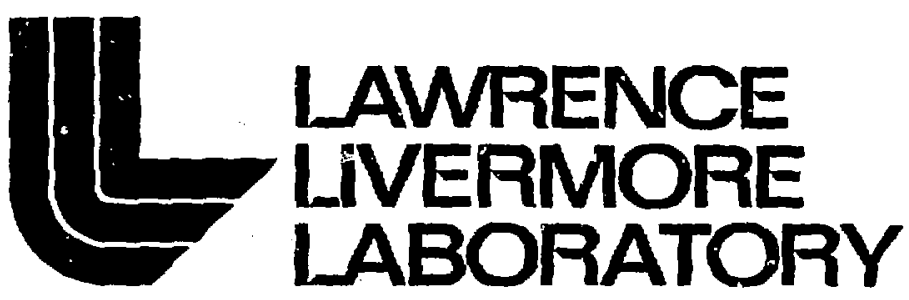

University of Califomia/Livermore

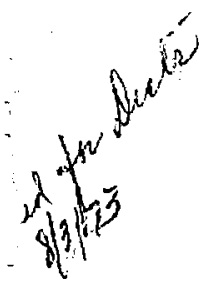

\section{MASTER}




\section{NOTICE}

"This report was prepatcd as an account of work sponsoted by the Unised States Government. Nelilier ihe Untied States not the United States Atomic Enardy Commisston, not any of thatr eniployecs, nor any of theit conteasions, subcoutractors, or theis employecs, makes any warraity, express of implied, of assumes

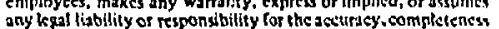
cr uscfulness of any informalion, apparatus, product or process disclored, ot tenesesss tiat les use would not infringe privatcly. owned atghts."

Printed in the United States of America Available from

National Technical Information Service

U.S. Department of Commerce 5285 Port Royal Road

Springfield, Virginia 22151

Price: Printed Copy $\$$ *; Microfiche $\$ 0.95$

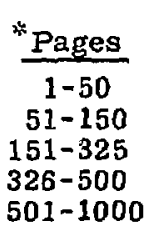

NTIS

Selling Price

$\$ 4.00$

$\$ 5.45$

$\$ 7.60$

$\$ 10.60$

$\$ 13.60$ 
TID-4500, UC-20

Controlled Thermonuclear

Procesaes

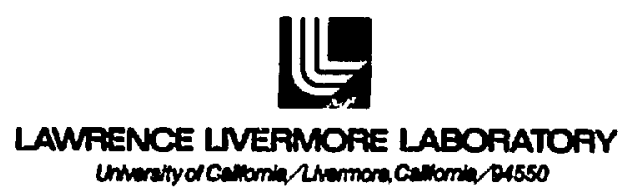

UCRL-51383

\section{A TWO-ZONE POTENTIAL RELAXATION SCHEME FOR HANDLING GRID WIRES}

R. R. Smith

S. L. Rompel

MS. date: June 1, 1973

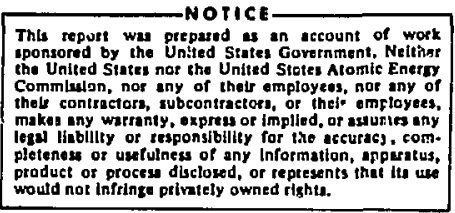

This repust wa prepased as an account of work eponsored by the United States Government. Netthes the United States not the United Stotes Alomic Enerry Commishon, nor sny of thest amployeas, nor any of thols contrectass, suconsractors, or thes omployses, logal linbillty of tesponsibility for the ateutacs, com. pletenoss of ustulness of any Information, apporatus, would not inftinte ptivelcly owned rights. 


\section{Contents}

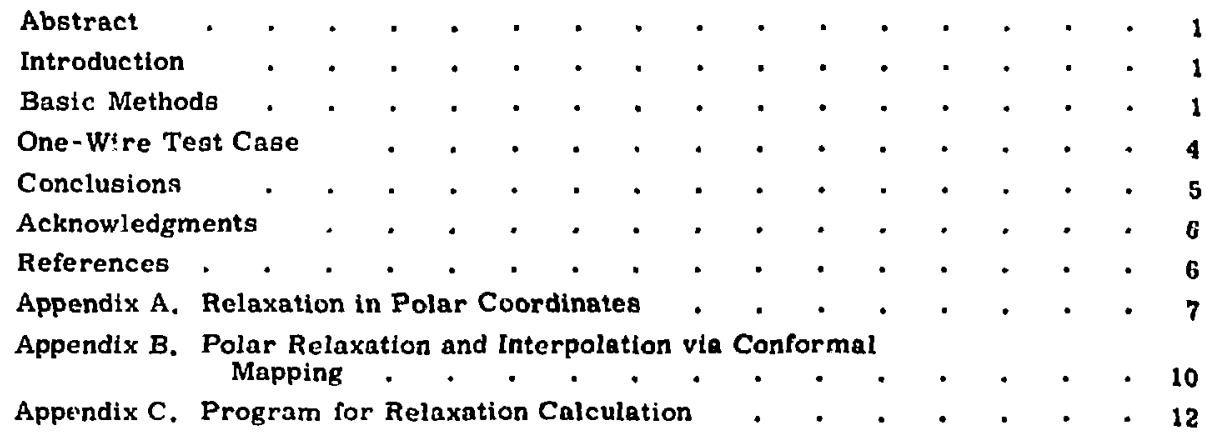




\title{
A TWO-ZONE POTENTIAL RELAXATION SCHEME FOR HANDLING GRID WIRES
}

\begin{abstract}
A rclaxation scteme for economically calculating two-dimensional electrostatic potentials in configurations with fine grid wires has been devised and tested. potentials calculated in this manner agree with potentials calculated with an analytic formula for the case of a one-wire test problem in which the radius of the wire is two orders of magnitude smaller than the outer boundary dimensions.

\section{Introduction}

The periodic-focusing direct-conversion unit ${ }^{1,2}$ and the venetian blind converte $\mathrm{r}^{3}$ both use grid wires which are quite small compared to other dimensions. There ar? several reasons why the flelds near the grid wires ars important. In both devices the electric field at the wires determines the likelihood of sparking and thus determines the maximum voltage which can be used. The exact potential distribution near the wircs of the periodic-focusing device has been shown to be unimportant to particle trajectories, because ions are moving rapidly there and small deflections do not prevent them from being captured in the collector cups. However, the venetlan blind device depends upon unfocused ballistic trajectories and is quite sensltive to defocusing effects. In that device the secondary electrons must be suppressed by grid wires close to the main collection electrodes. These grid wires are in a geometry which prevents easy estimation of the potentials. Potentials in complicated geometries are usually calculated by a relaxation methoc. This method is eccurate only if the mesh size is comparable to the smallest scale length of the potential. For direct conversion work this length is the radius of the wire, which can be $10^{3}$ times smaller than the other dimensions of interest. Calculations based on uniform subdivisions of such small size would be prohibitively costly in computer time. Instead we combine finely divided regions near the wires with coarse zoning away from the wires.
\end{abstract}

\section{Basic Methods}

We shall consider two-dimensional configurations, such as shown in Figs. 1 and 2. The large-scale potential distributions have rectangular geometry, and the 


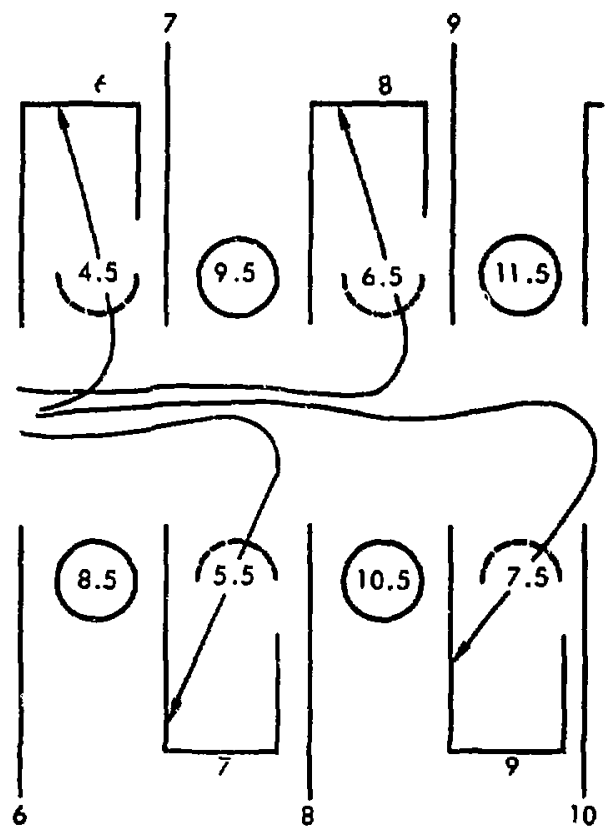

Fig. 1. Cross-sectional segment of the periodic-focusing collector, showing relative potentials and typical ion orbits. Low-potential grids are shown as dotted circles and high-potential rods as solid circles.

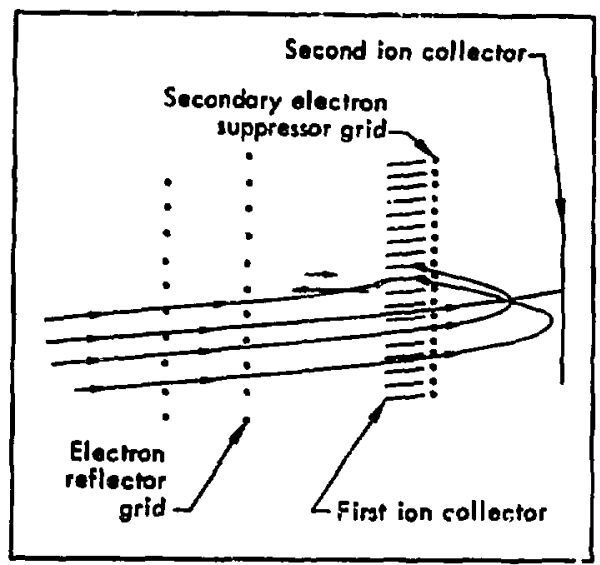

Fig. 2. Detall of two-gtage venetian blind converter.

coarse-zoned regions are divided into rectangular cells of dimension $\Delta x$ by $\Delta y$. At each point $(1, j)$ the potential is iterated according to the formula,

$$
\begin{aligned}
& \phi_{i, j}^{N}=(1-\alpha)_{\phi_{i, j}^{N}-1}^{N}+\frac{(\Delta x)^{2}(\Delta y)^{2}}{(\Delta x)^{2}+(\Delta y)^{2}} \frac{\alpha}{2} \\
& \times\left[\frac{\phi_{i, j-1}^{N}+\phi_{i, j+1}^{N-1}}{(\Delta y)^{2}}+\frac{\phi_{i-1, j}^{N}+\phi_{i+1, j}^{N-1}}{(\Delta x)^{2}}\right],
\end{aligned}
$$

where $\mathbf{N}$ is the present iteration, $\mathbf{N}-1$ is the previous iteration, and $\alpha$ is the so-called over-relaxation parameter. ${ }^{4}$ This parameter is typically about 1.7 , and its use greatly speeds the convergence. Too large a value for $\alpha$ can result in instability. The relaxation pattern alternates, going from left to right and top to bottom on odd $\mathbf{N}$ and right to left and bottom to top on even $\mathrm{N}$.

As rnentiuned above, it is impractical to calculate the potentials near grid wired by rectangular relaxation, since the meshes would have to be approximately equal to the wire in size. It is desirable to haye a fine scale cylindrical mesh centered on eact. wire which would be connected in some way with the coarse rectangular mesh.

As shown in Appendix $A$, if one choosfe the $n^{\text {th }}$ mesh radius to be $r_{n}=2^{n} r_{0}$ and $\Delta \theta=2 \pi / \mathrm{p}$, where $\mathrm{p}$ is an integer, then a relaxation formula for the new value of $\phi$ at $\theta=m \Delta \theta, r=r_{n}$ in terms of the previous surrounding $\phi$ values is 
$\left.\phi_{n}^{m}=\frac{10}{3}\left[\frac{1}{\left(\frac{q}{\pi}\right)^{2}+6}\right] \phi_{n+1}^{m}+\frac{8}{3}\left[\frac{1}{\left(\frac{p}{\pi}\right)^{2}+6}\right] \phi_{n-1}^{m}+\frac{1}{2}\left[\frac{1}{1+6\left(\frac{\pi}{p}\right)^{2}}\right]^{m-1}+\phi_{n}^{m+1}\right)$

An alternative relaxation formula

$$
\phi_{n}^{m}=\frac{\phi_{n-1}^{m}+\phi_{n+1}^{m}}{2\left[1+\left(\frac{p}{2 \pi}\right)^{2}(l n 2)^{2}\right]}+\frac{\phi_{n}^{m-1}+\phi_{n}^{m+1}}{2\left[1+\left(\frac{2 \pi}{p}\right)^{2}\left(\frac{1}{I n 2}\right)^{2}\right]} .
$$

is derived in Appendix $B$.

The second formula [Eq. (3)] seems to have better limiting beharior than the first, although nu flaw in the derivation of Eq. (2) is apparent to the authors.

In order to implement the overlapping of the two relaxation zones, one might proceed as follows:

1. Specify the potentials on the rectangular mesh boundary and also on the grid wires; for example, points 1, 2, 3 on Fig. 3.

2. Relax the potential on the rectangular mesh, ignoring the wires.

3. From the $\phi$ values on the rectangular mesh, interpolate to get the potentials on the outer radius of the polar zone at each wire, such as points 4 and 5 .

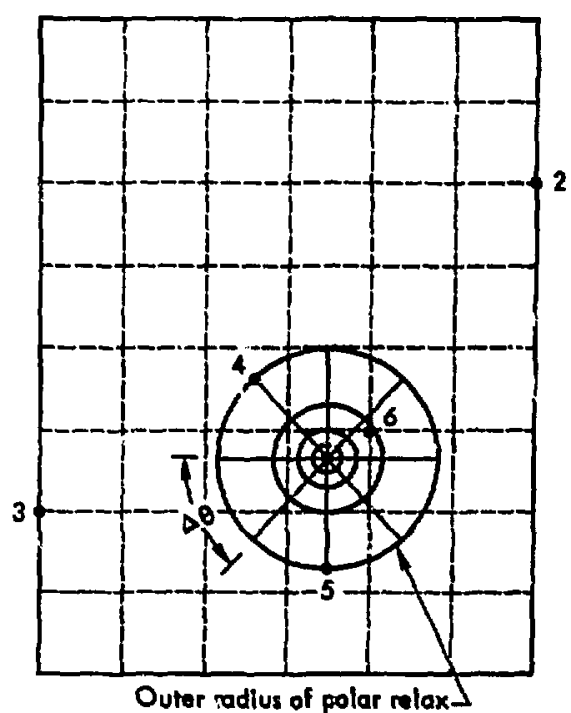

Fig. 3. Overlapping of rectangular and variable-radii polar relaxation zones. The grio wire is at point 1.
4. Holding $\phi$ fixed at points 4,5 . etc., to the polar relaxation. This is where the grid potential is felt.

5. Now from the polar $\phi$ values, interpolate as in Appendix B to find $\phi$ on the rectangular mesh points which lie within the polar region and are nearest the wire, such as point 6 .

6. Holding $\phi$ fixed at points auch as 6 , return to step 2 and repeat the process.

Once the potentials are computed for the square and polar meshes, for each $(x, y)$ location, one must agk whether there is a grid wire nearby, and if 00 , which polar table must be used to compute $\phi$.

For the rectangular mesh, $\Delta x$ and $\Delta y$ need not be the same. Since troutic is anticipated if the wire is too close to 
a rectangular mesh point, the wires should be located roighly in the center of a mesh rectangle. A plausible choice for the radius of the polar repion is somewhat larger than two rect... gular mesh spacings.

\section{One-Wire Test Case}

The above two-zone relaxation scheme was tested in the case of a planar grid triode. Spangenberg ${ }^{5}$ gives an approximate but accurate expression for the potential in a configuration of evenly spaced grid wires between two parallel plates. Figure 4

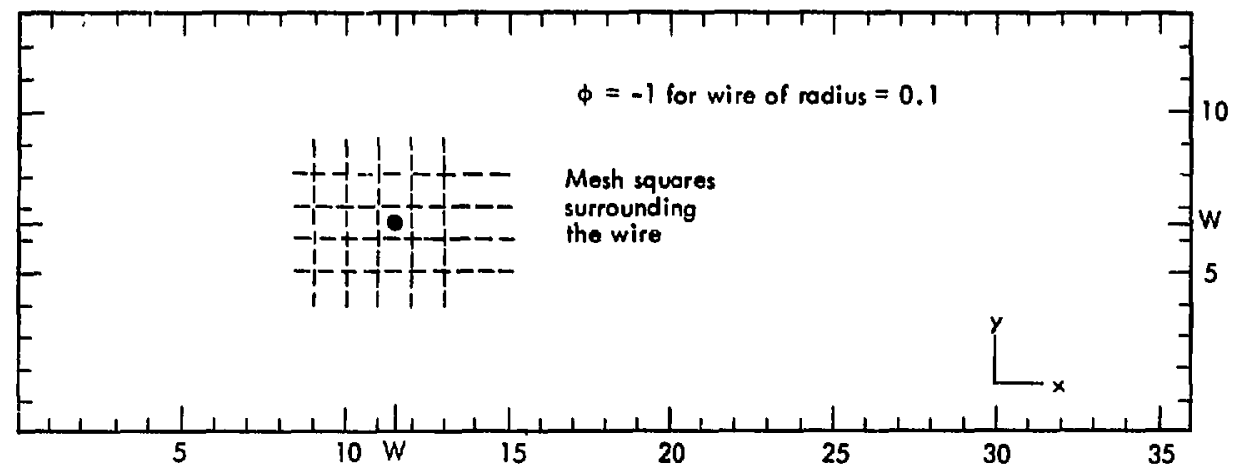

Fig. 4. Geometry $c$ ? one-wire test problem. The boundary conditions are $\phi=0$ and $\phi=2$.

shows the $13 \times 36$ square mesh used in the test problem. The fixed boundary potentials are zero on the left edge, 2 on the edge $x=36$, and -1 at the wire of radius 0.1 located at $(11.5,6.5)$. The potentials on the top and bottom boundaries are specified by the analytic expression for the potential, which is

$\phi(x, y)=K_{1} \ln \left\{e^{\frac{4 \pi \dot{\alpha}}{a}\left(x-d_{1}\right)}+1-2 \cos \left[\frac{2 \pi d_{2}}{a}\left(y-\frac{a}{2}\right)\right] e^{\frac{2 \pi d_{2}}{a}\left(x-d_{1}\right)}\right\}+K_{2} x$

where $d_{1}=11.5, d_{2}=24.5, a=13, K_{1}=0.1203$, and $K_{2}=-0.02357$ for the present geometry and potentials.

The potentials obtained from the analytic formula are compared with the numbers obtained from the relaxation scheme in Table 1. Only the numbers on the line $x=11$ which passes close to the wire are shown. There are two columns of relaxed potentials. Sixteen angular divisions were used. The numbers listed under $\phi_{\text {equal }}$ are obtained with equally spaced radii of $0.1,0.404,0.707,1.011,1.314,1.618,1.921$, and 2.225 . The numbers under $\phi_{\text {varied }}$ were obtained with the relaxation formula [Eq. (2)], using 
Table 1. Comparison of analytic and relaxed potentials at $x=11$.

\begin{tabular}{cccc}
\hline$y$ & Qnalytic & Q.zqual & Qvaried \\
\hline 0 & -0.119817 & -0.119817 & -0.119817 \\
1 & -0.126810 & -0.130345 & -0.127022 \\
2 & -0.148615 & -0.155823 & -0.148968 \\
3 & -0.188162 & -0.199562 & -0.188693 \\
4 & -0.252294 & -0.268799 & -0.252986 \\
5 & -0.357746 & -0.379900 & -0.357338 \\
6 & -0.546669 & -0.574233 & -0.541065 \\
7 & -0.546669 & -0.574235 & -0.541066 \\
8 & -0.357746 & -0.379901 & -0.357338 \\
9 & -0.252291 & -0.268801 & -0.252986 \\
10 & -0.138162 & -0.199565 & -0.188693 \\
11 & -0.148615 & -0.155826 & -0.148968 \\
1. & -0.126810 & -0.130347 & -0.127022 \\
13 & -0.119817 & -0.119817 & -0.119017 \\
\hline
\end{tabular}

mesh radii of $0.1,0.2,0.4,0.8,1.6$, and 3.2 . The numbers obtained with varlable mesh spacing agree quite well with the semi-exact analytic values. The error seems to be about $1 \%$ and is on the correct side of the "true" answer, i.e., the relaxed values underestimate the effect of the wre. The relaxed numbers are nearly symmetric with respect to the line $y=6-1 / 2$, as they must be.

\section{Conclusions}

The :wo-zone relaxation method for handling grid wires in two-dimensional electrostatic potential problems appears to work quite well, and a progressive increase of mesh radius at greater distances from the wires is a considerable improvement over a constant mesh radius. It is intuitively clear that the polar zones should not overlap each other or a boundary. We speculate that over-relaxing is much more important in the coarse rectangular mesh than in the polar meshes, because the former involve many more mesh points.

An additional test of the method would be the calculation of potentials near the electron suppression grids of a venetian blind collector. These potentials have been measured on resistance paper by Phil Maslin. 


\section{Acknowledgments}

The vuthors would like to thank Ralph Moir and Bill Barr for help in the direction and presentation of this woak and also Don Moore and Dave Faul, who worked on the relaxation method of Apgendix A.

\section{References}

1. R.F. Post, in Proc. Int. Conf. on Nuclear Fusion Reactors (Culham Laboraiory, Culham, England, September 17-19, 1969), Pp. 88-111.

2. R. W, Moir, W. L. Barr, R. P. Frels, and R.F. Post. Plasma Physics and Co,1trolled Nuclear Fusion Research III. pp. 315-328 (1971).

3. R.W. Moir and W. L. Barr, Nucl. Fusion 13, 35 (1973).

4. P.T. Kirstein, G.S. Kino, and W.E. Waters, Space-Charge Flow (McGraw Hils Book Co., New York, 1967), p. 433.

5. K. R. Spangenberg, Vacuum Tubes (McGraw Hill Book Co., New York, 1948), p. 125. 


\section{Appendix A \\ Relaxation in Polar Coordinates}

This is an annotation of work done by Don Moore (December 1971). In Polar coordinates, Laplace's equation is

$$
\nabla_{\phi}^{2}=\frac{\partial^{2} \phi}{\partial r^{2}}+\frac{1}{r} \frac{\partial \phi}{\partial r}+\frac{1}{r^{2}} \frac{\partial^{2} \phi}{\partial r^{2}}
$$

If the notation of Fig. A-1 is used, the difference equation is

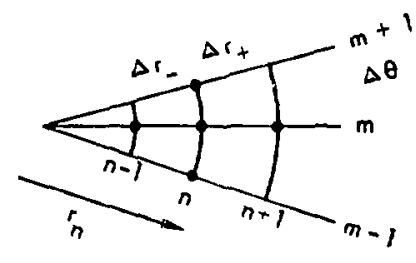

Fig. A-1. Notation used in the development of Eq. (2).

$$
\begin{gathered}
\frac{\phi_{n+1}^{m}-2 \phi_{n}^{m}+\phi_{n-1}^{m}}{(\Delta r)^{2}}+\frac{1}{r_{n}} \frac{\phi_{n+1}^{m}-\phi_{n-1}^{m}}{2 \Delta r} \\
+\frac{1}{r_{n}^{2}} \frac{\phi_{n}^{m+1}-8 \phi_{n}^{m}+\phi_{n}^{m-1}}{(\Delta \theta)^{2}}=0 .
\end{gathered}
$$

This is true when the radial increments are equal. When they are unequal, we will resort to a Taylor expanston in $r$

to obtain $\partial^{2} \phi / \partial r^{\partial}$ and $\partial \phi / \partial r$. The expansions are

$$
\phi\left(r+\Delta r_{+}, \theta\right)=\phi(r, \theta)+\frac{\partial \phi}{\partial r} \Delta r_{+}+\frac{\partial^{2} \phi}{b r^{2}} \frac{\left(\Delta r_{+}\right)^{2}}{2}+\ldots
$$

and

$$
\phi\left(r-\Delta r_{-}, \theta\right)=\phi(r, \theta)-\frac{\partial \phi}{\partial r} \Delta r_{-}+\frac{\partial^{2} \phi}{\partial r^{2}} \frac{\left(\Delta r_{-}\right)^{2}}{2}-\ldots
$$

By eliminating $\partial_{\phi} / \partial r$ we find

$$
\frac{\partial^{2} \phi_{n}^{m}}{\Delta r^{2}}=\frac{2 \phi_{n+1}^{m}}{\Delta r_{+}\left(\Delta r_{-}+\Delta r_{+}\right)}-\frac{2 \phi_{n}^{m}}{\Delta r_{+} \Delta r_{-}}+\frac{2 \phi_{n-1}^{m}}{\Delta r_{-}\left(\Delta r_{-}+\Delta r_{+}\right)^{\prime}} .
$$

Similarly one can eliminate $\partial^{2} \phi / \partial r^{2}$ from Eqs. $(A-3)$ and $(A-4)$ to obtain

$$
\frac{\partial \phi_{n}^{l n}}{\partial r}=\frac{\Delta r_{-} \phi_{n+1}^{m}}{\Delta r_{+}\left(\Delta r_{-}+\Delta r_{+}\right)}+\frac{\left(\Delta r_{+}-\Delta r_{-}\right)_{n}^{m}}{\Delta r_{+} \Delta r_{-}}-\frac{\Delta r_{+} \phi_{n-1}^{m}}{\Delta r_{-}\left(\Delta r_{-}+\Delta r_{+}\right)} .
$$


Suprose the $n^{\text {th }}$ grid has radius $I_{n}$, where

$$
r_{n}=r_{0}+\Delta r \frac{K^{n}-1}{K-1}
$$

Thus $\Delta r_{+}=K^{n} \Delta r, \Delta r_{-}=K^{n-1} \Delta r$, where $K$ is a constant. Substituting into Eqs. (A-5) and $(A-6)$, we find

$$
\frac{\partial \phi_{n}^{m}}{\partial r}=\frac{\phi_{n+1}^{m}}{K^{n}(K+1) \Delta r}+\frac{(K-1) \phi_{n}^{m}}{K^{n} \Delta r}-\frac{K^{2} \phi_{n-1}^{m}}{K^{n}(K+1) \Delta r}
$$

and

$$
\frac{\partial^{2} \phi_{n}^{m}}{\theta r^{2}}=\frac{2 K \phi_{n+1}^{m}}{K^{2 n}(K+1)(\Delta r)^{2}}-\frac{2 K \phi_{n}^{m}}{K^{2 n}(\Delta r)^{2}}+\frac{2 K^{2} \phi_{n-1}^{m}}{K^{2 n}(K+1)(\Delta r)^{2}} .
$$

Substituting into Eq. $(A-1)$, with $\Delta \theta=2 \pi / p$, where $p$ is an integer

$$
\begin{aligned}
& {\left[\frac{2 K r_{n}^{2}}{K^{2 n}(K+1)(\Delta r)^{2}}+\frac{r_{n}}{K^{n}(K+1) \Delta r}\right] \phi_{n+1}^{m}+\left[\frac{(K-1) r}{K^{n} \Delta r}-\frac{2 K r_{n}^{2}}{K^{2 n} \Delta r^{2}}\right] \phi_{n}^{m}} \\
& +\left[\frac{2 K^{2} r_{n}^{2}}{K^{2 n}(K+1)(\Delta r)^{2}}-\frac{K^{2} r_{n}}{K^{n}(K+1) \Delta r}\right] \phi_{n-1}^{m}+\frac{p^{2}}{4 \pi^{2}}\left[\phi_{n}^{m+1}-2 \phi_{n}^{m}+\phi_{n}^{m-1}\right]=0
\end{aligned}
$$

Now let $r_{0}=\Delta r$, so that $r_{n}=\Delta r \frac{K^{n}+K-2}{K-1}, n=0,1,2 \ldots$ One abtains

$$
\phi_{n}^{m}=a_{n} \phi_{n+1}^{m}+b_{n} \phi_{n-1}^{m}+c_{n} \phi_{n}^{m+1}+d_{n} \phi_{n}^{m-1} .
$$

where

$$
a_{n}=\frac{c_{+}(n)}{c_{0}(n)}, \quad b_{n}=\frac{c_{-}(n)}{c_{0}(n)}, \quad c_{n}=d_{n}=\frac{c(n)}{c_{0}(n)},
$$

and

$$
c_{+}(n)=\frac{3 k^{n+1}-k^{n}+2 K^{2}-4 K}{k^{2 n+2}-K^{2 n}} .
$$




$$
\begin{aligned}
& c_{-}(n)=\frac{3 K^{n+2}-K^{n+3}+2 K^{3}-4 K^{2}}{K^{2 n+2}-K^{2 n}}, \\
& c(n)=\frac{(K-1) p^{2}}{4 \pi^{2}\left(K^{n}+K-2\right)},
\end{aligned}
$$

and

$$
c_{0}(n)=\frac{2 p^{2} K^{2 n}(K-1)^{2}+8 \pi^{2} K\left(K^{n}+K-2\right)^{2}-4 \pi^{2} K^{n}(K-1)^{2}\left(K^{n}+K-2\right)}{4 \pi^{2}\left(K^{n}+K-2\right) K^{2 n}(K-1)}
$$

In the special case $r_{n}=2^{n} r_{0}, \Delta \theta=2 \pi / p$, we have Eq. (2),

$$
\phi_{n}^{m}=\frac{10}{3}\left[\frac{1}{\left(\frac{p}{\pi}\right)^{2}+6}\right] \phi_{n+1}^{m}+\frac{8}{3}\left[\frac{1}{\left(\frac{p}{\pi}\right)^{2}+6}\right] \phi_{n-1}^{m}+\frac{1}{2}\left[\frac{1}{1+6\left(\frac{\pi}{p}\right)^{2}}\right]\left(\phi_{n}^{m+1}+\phi_{n}^{m-1}\right) .
$$

In circular symmetry, where $\phi_{n}^{m+1}=\phi_{n}^{m}$, this reduces to

$$
\phi_{n}^{m}=\frac{5}{9} \phi_{n+1}^{m}+\frac{4}{9} \phi_{n-1}^{m} .
$$

This is independent of $p$, as it. should be, but does not seem to reproducc a logarithmic variation in potential between two concentric cylinders. 


\section{Appendix B \\ Polar Relaxation and Interpolation via Conformal Mapping}

Suppose we use conformal mapping to transform a pie-wedge into a rectangle (Fig. B-1).

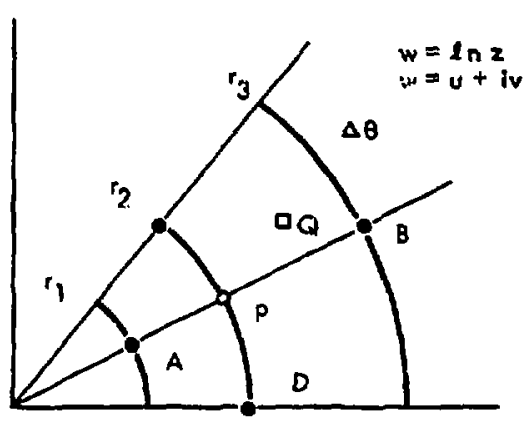

(a)

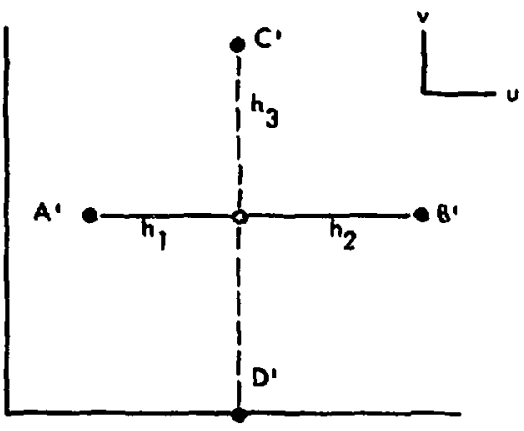

(b)

Fig. B-1. Notation used in the development of Eq. (3).

We wish to expand the potentials at $B^{\prime}$ and $A^{\prime}$ in Taylor series and eliminate $\theta_{\phi} / \partial u$ in analogy with Appendix A. This time we have transformed away the first derivattve from the Laplacian. Dropping the primes for convenience,

$$
\phi_{B}=\phi_{p}+\frac{\partial \phi}{\partial u} h_{2}+\frac{1}{2}\left(\frac{\partial^{2}}{\partial u^{2}} h_{2}^{2}\right)+\ldots
$$

and

$$
\phi_{A}=\phi_{p}-\frac{\partial \phi}{\partial u} h_{1}+\frac{1}{2}\left(\frac{\partial^{2} \phi_{\phi}^{2}}{\partial u^{2}} h_{1}^{2}\right)+\ldots
$$

Eliminating $\partial_{\phi} / \partial u$ we find

$$
\frac{\partial^{2} \phi_{p}}{\partial u^{2}}=\frac{2\left(h_{1} \phi_{B}+h_{2} \phi_{A}\right)}{h_{1} h_{2}\left(h_{1}+h_{2}\right)}-\frac{2}{h_{1} h_{2}} \phi_{p}
$$

Similarly 


$$
\frac{\partial^{2} \phi_{p}}{\partial v^{2}}=\frac{1}{h_{3}^{2}}\left(\phi_{C}+\phi_{D}\right)-\frac{2}{h_{3}^{2}} \phi_{p}
$$

Substituting into $\partial^{2} \phi / \partial u^{2}+\partial^{2} \phi / \partial u^{2}=0$, we obtain

$$
\phi_{P}=\frac{h_{1} h_{2}\left(\phi_{C}+\phi_{D}\right)+\frac{2 h_{3}^{2}}{h_{1}+h_{2}}\left(h_{1} \phi_{B}+h_{2} \phi_{A}\right)}{2\left(h_{1} h_{2}+h_{3}^{2}\right)} .
$$

This can be checked in various limits, If the mesh to square, $h_{1}=h_{2}=h_{3}=h_{4}$ and one obtains the usual result,

$$
\phi_{P}=\frac{1}{4}\left(\phi_{A}+\phi_{B}+\phi_{C}+\phi_{D}\right)
$$

If the problem is one-dimensional or circularly symmetric, $\phi_{C}=\phi_{D}=\phi_{p}$ and Eq. (B-5) reduces to

$$
\phi_{p}=\frac{h_{1} \phi_{B}+h_{2} \phi_{A}}{h_{1}+h_{2}}=\frac{\ln \frac{r_{2}}{r_{1}} \phi_{B}+\ln \frac{r_{3}}{r_{2}} \phi_{A}}{\ln \left(r_{3} / r_{1}\right)} .
$$

This is, of course, the exact answer for the potential between two concentric cylindera when $r_{2}$ is considered to be variable, and it is independent of $h_{3}$. The limit $\epsilon \rightarrow 0$ of $r_{3} / r_{2}=1+\epsilon=r_{2} / r_{1}$ and $\Delta \theta=\epsilon$ is also correct in this second treatment.

If we specialize to a variable mesh grid wire problem where $r_{n}=r_{0} 2^{n}$ and $\Delta \theta=2 \pi / p$, then $h_{1}=\ell n 2=h_{2}$, and $h_{3}=\Delta \theta$. We obtain Eq. (3)

$$
\phi_{n}^{m}=\frac{\phi_{n-1}^{m}+\phi_{n+1}^{m}}{2\left[1+\left(\frac{p}{2 \pi}\right)^{2}(l n 2)^{2}\right]}+\frac{\phi_{n}^{m-1}+\phi_{n}^{m+1}}{2\left[1+\left(\frac{2 \pi}{p}\right)^{2}\left(\frac{1}{l n 2}\right)^{2}\right]},
$$

where the notation of Appendix A, $(\ell n 2)^{2}=0.4805$, is used.

The coefficients are different from those of Appendix $A$, and the latter do not seem to reduce to the correct answers in the limits of $1 \mathrm{D}$ or circular symmetry, yet the fault is not apparent to the authors.

The above transformation is also useful in interpolating from the known potentials at points $A, B, C, D$ to that at point $Q$ on Fig. $B-1$. 


\section{Appendix C \\ Program for Relazation Calculation}

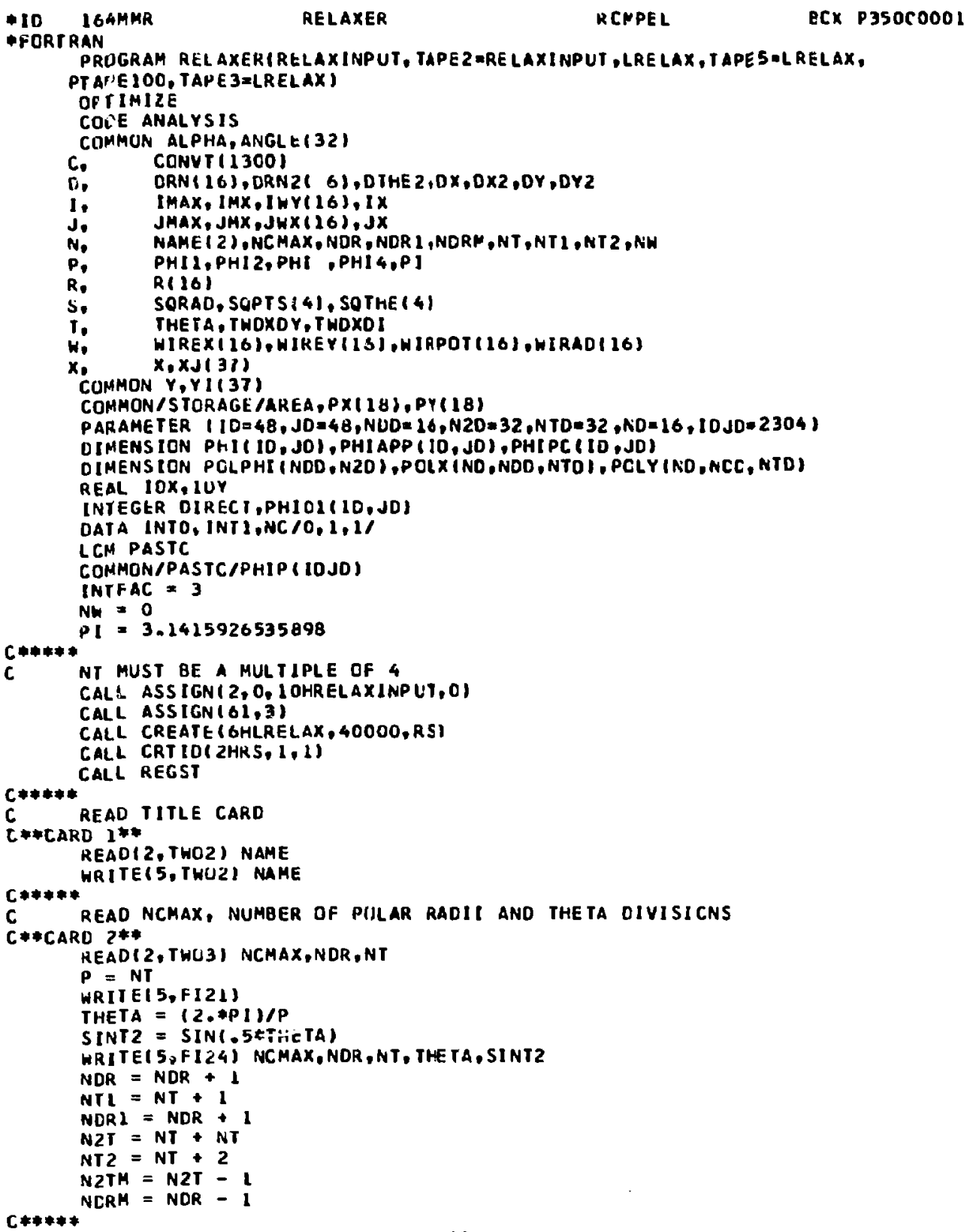




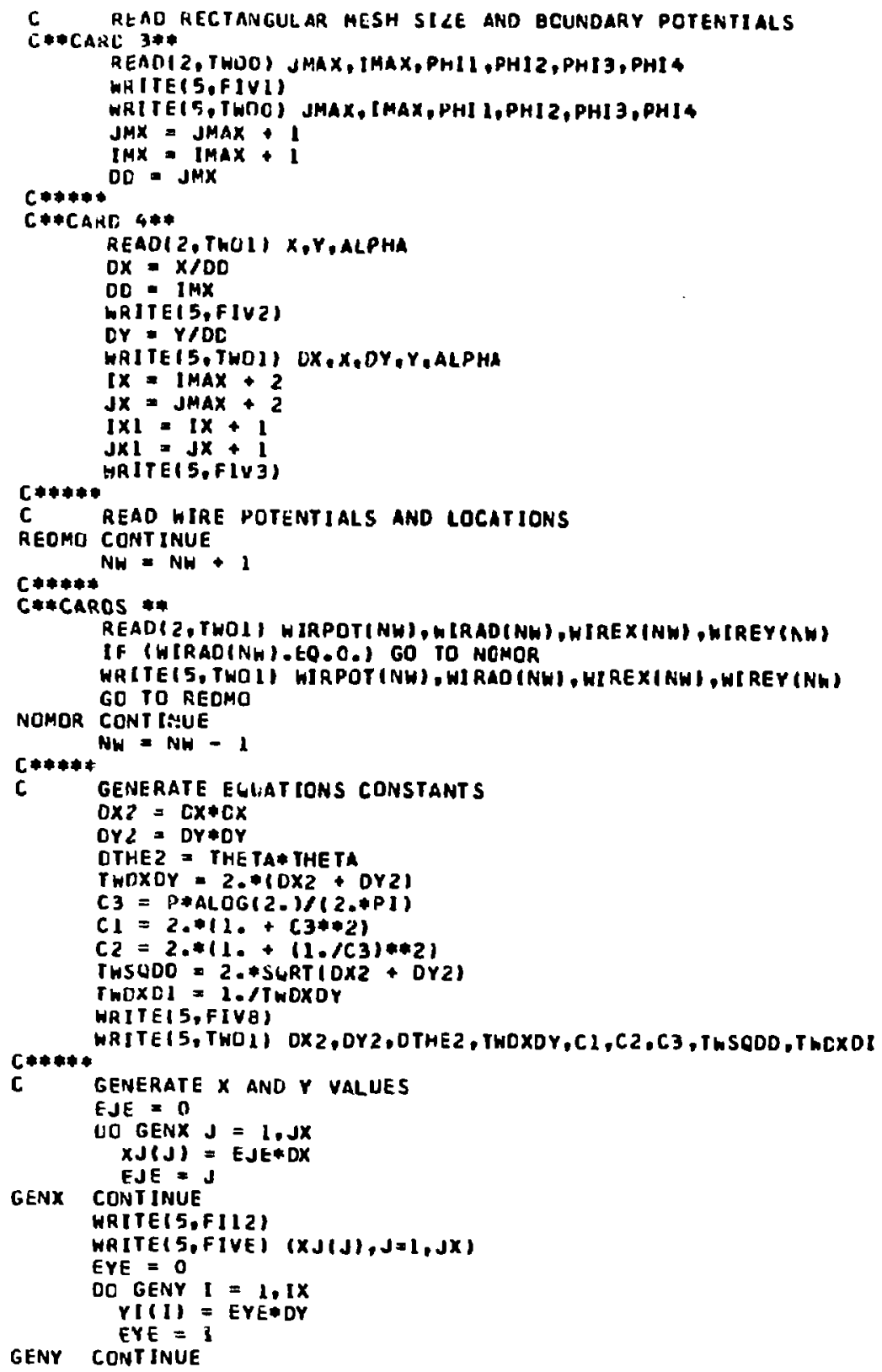




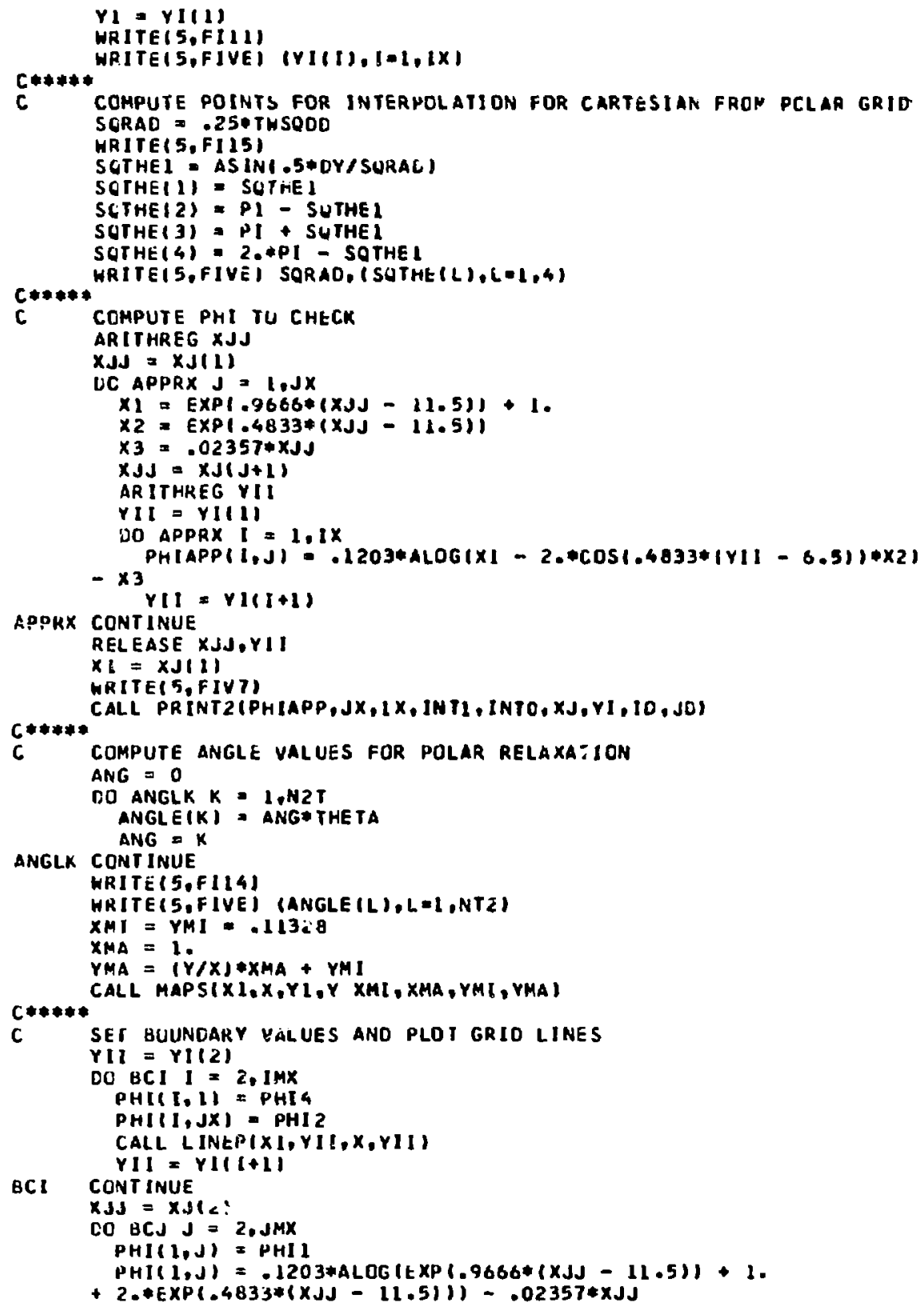




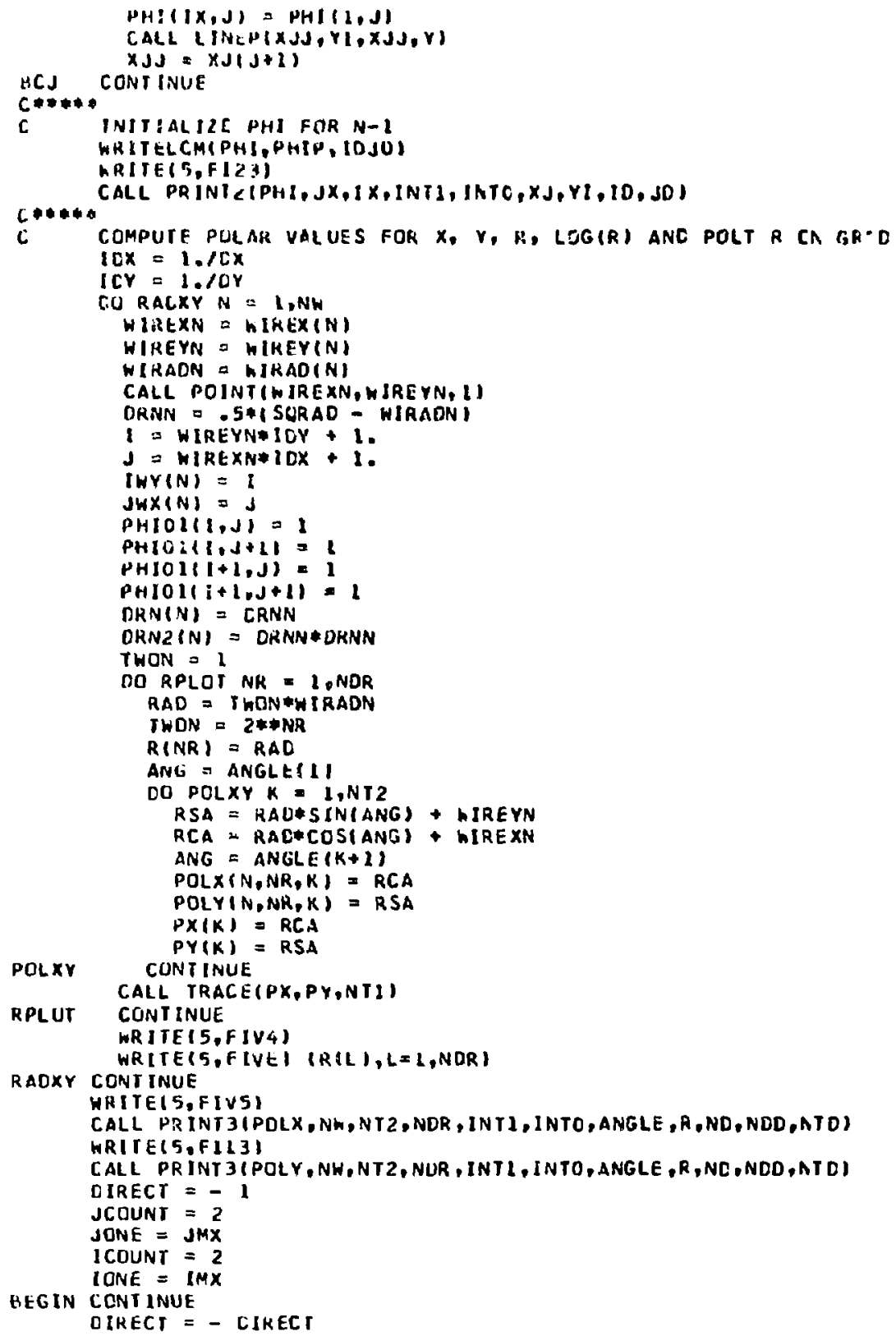




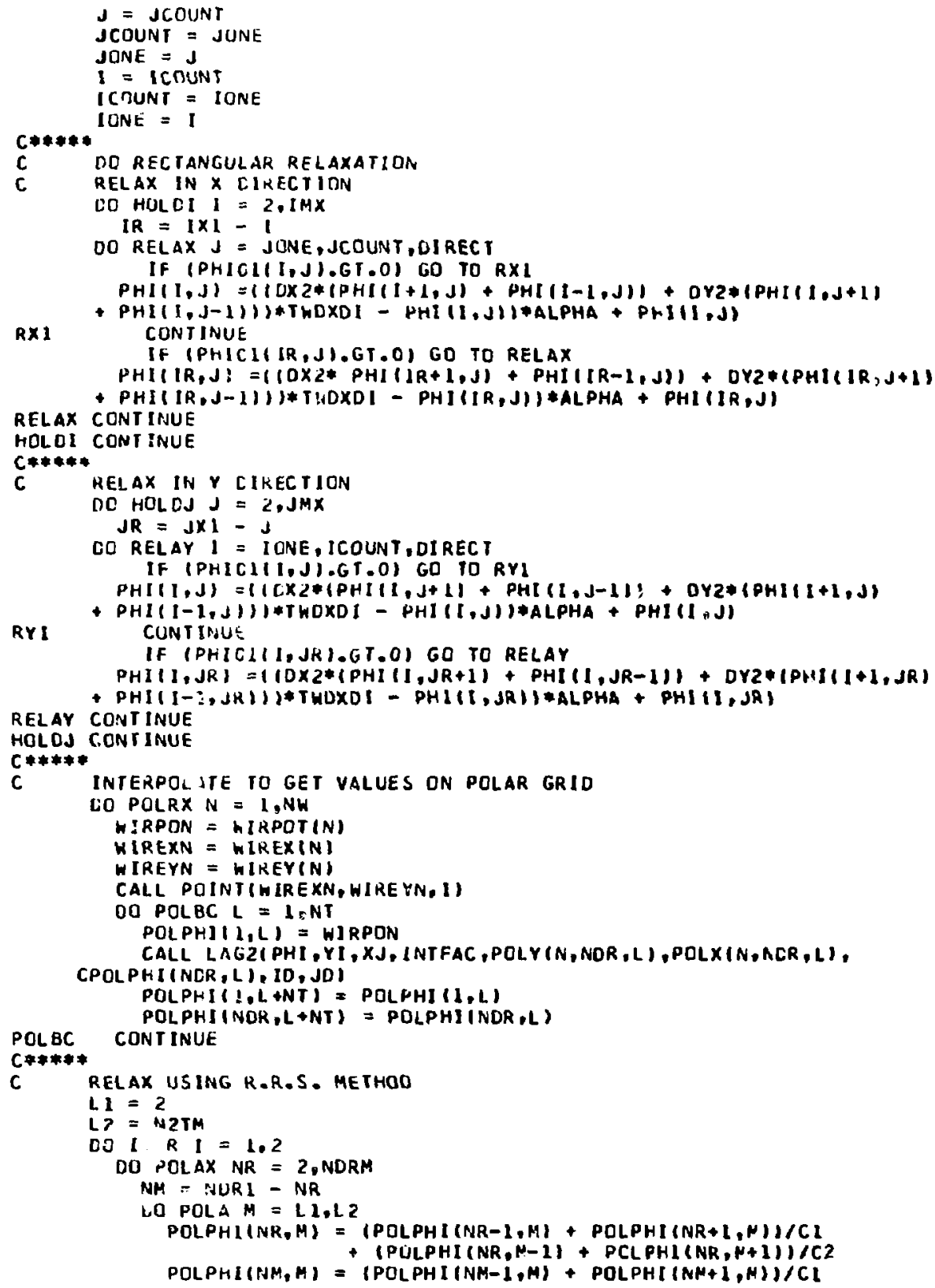




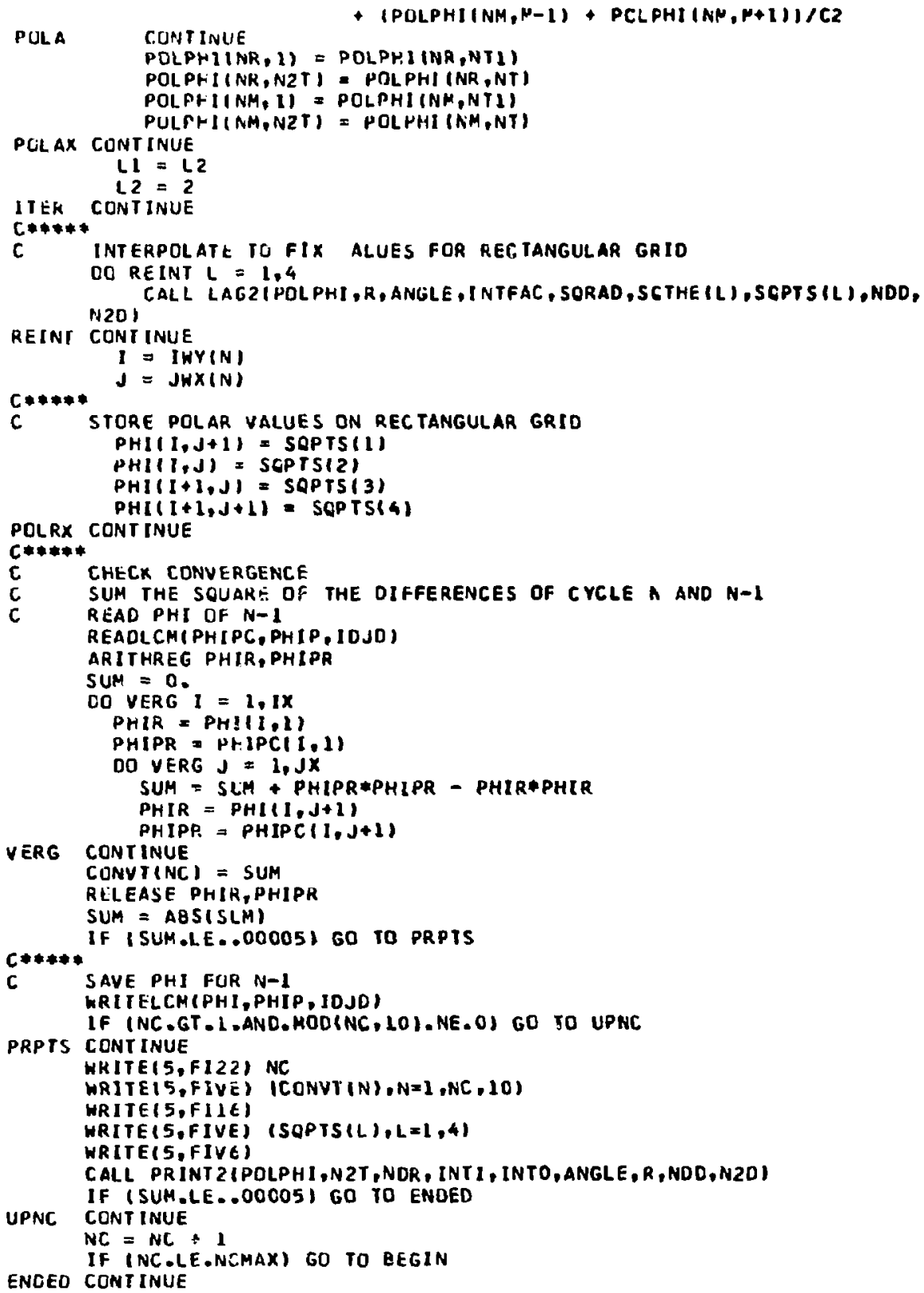




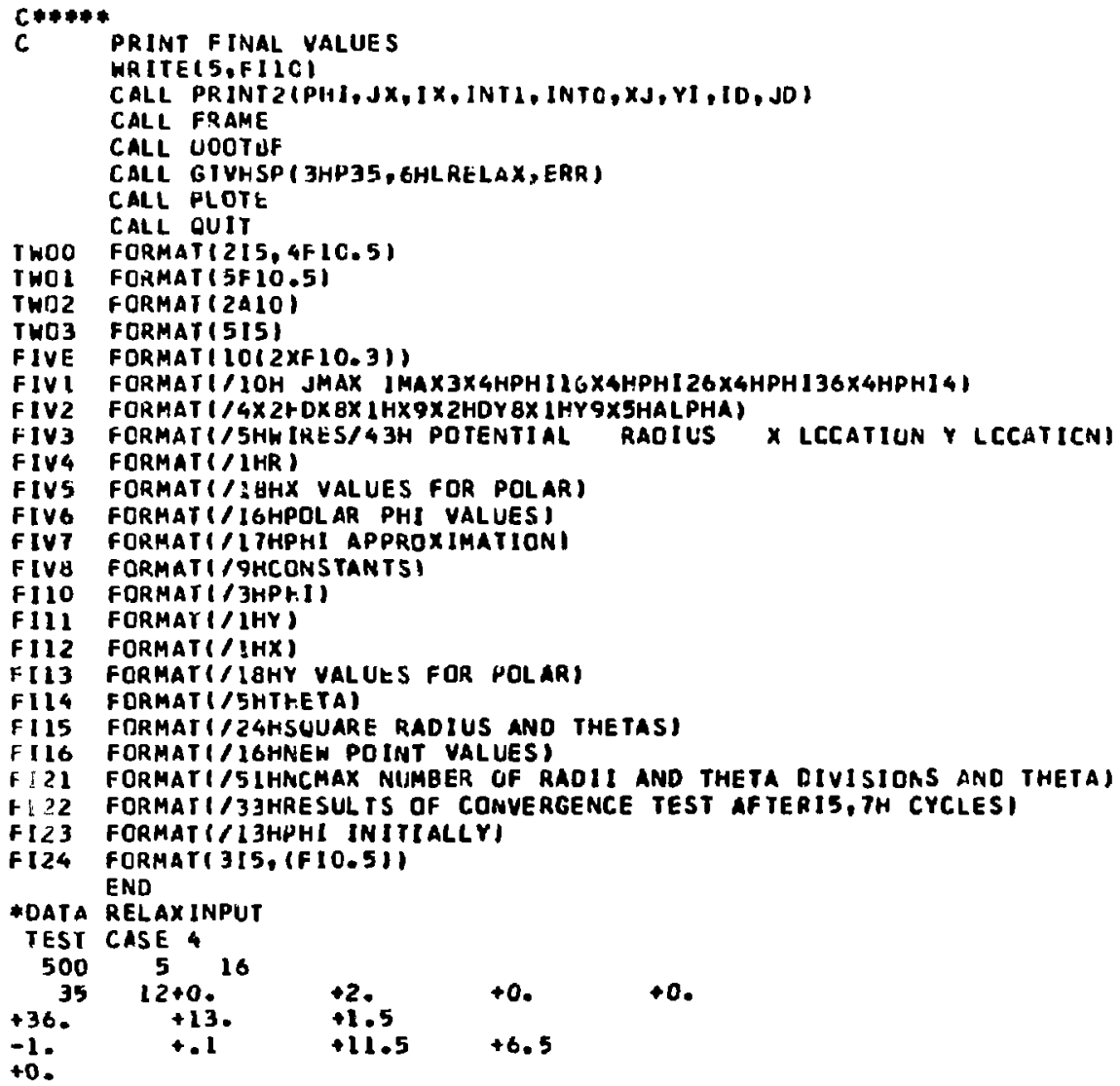

